\title{
Multiple Sclerosis and Ultraviolet Radiation: Time to Shed More Light
}

\author{
A.J. McMichael A.J. Hall
}

Multiple sclerosis (MS) has tantalised epidemiologists for half a century. The incidence is consistently higher in females than in males. In general, rates increase with latitude, although there are some noteworthy exceptions to this geographic gradient. Populations migrating between regions of discrepant risk tend to carry their original risk with them if they migrate after adolescence - but not if they migrate early in life, in which case they acquire the risk of their host environment population.

Many risk factors have been invoked for MS. These include genetic susceptibility, dietary lipids, certain physicochemical (e.g. solvent) exposures, unusual patterns of childhood viral infectious disease, glandular fever and (reduced) exposure to ultraviolet radiation (UVR). As epidemiological studies have proceeded - comprising descriptive studies over time and place, migrant studies, and, less often, case-control and cohort studies - the pathogenesis of the disease has gradually become clearer. It is now generally agreed that MS entails an autoimmune process that destroys basic myelin protein (and hence blocks conduction) $[1,2]$.

That autoimmune basis to the disease may facilitate the drawing together of several of the otherwise apparently disparate risk factors. Viral infections, especially those occurring in later childhood and adolescence, can induce self-attack - that is, autoimmunity - when the immune system fails to distinguish between viral protein and myelin protein. The similarity between peptides, of course, is not accidental; it is via this molecular mimicry process that the virus enhances its survival and replication prospects. There is considerable evidence that infection with the Epstein-Barr virus in particular is involved in initiating MS [3-5]. The resultant autoimmune process is presumably modulated by certain factors that have immune-suppressive activity, including dietary polyunsaturated fats (especially the n-3 polyunsaturates, prominent within fish oils) and UVR.

That last-mentioned factor, UVR, appears as subject of overlap in the two epidemiological studies of MS published in this issue of the journal. From much animal experimental research and some observations in human studies, it is apparent that UVR exposure has an immunesuppressive effect. The details are not fully resolved, but it seems to encompass both local and systemic immune suppression, including via suppression of Th1 cell activity. Since these cells mediate the attack on 'self' that characterises MS, and some other autoimmune diseases, it is plausible that UVR influences some aspect of MS pathogenesis. There are other possibilities too: UVR increases the synthesis of vitamin $\mathrm{D}_{3}$, and that in turn has a known immune-suppressive action. Indeed, a Japanese study has reported that a polymorphism (specifically the $b$ allele) in

\begin{tabular}{ll}
\hline KARGER & ( 2001 S. Karger AG, Basel \\
Fax +4161306 1234 & 0251-5350/01/0203-0165\$17.50/0 \\
$\begin{array}{l}\text { E-Mail karger@karger.ch } \\
\text { www.karger.com }\end{array}$ & $\begin{array}{l}\text { Accessible online at: } \\
\text { www.karger.com/journals/ned }\end{array}$
\end{tabular}

Prof. A.J. McMichael, Professor of Epidemiology Department of Epidemiology and Population Health London School of Hygiene and Tropical Medicine Keppel Street, London WC1E 7HT (UK)

Tel. +44 207612 7825, Fax +44 207580 6897, E-Mail tony.mcmichael@lshtm.ac.uk 
the vitamin D receptor gene is associated with the occurrence of MS [6].

That much is relatively easy to agree. As the paper by van der Mei and colleagues makes clear, the remarkably strong geographic gradient of MS prevalence, state by state in Australia, is most strongly correlated with ambient UVR levels (although this ambient exposure, in turn, may be confounded by geographic variations in sunrelated outdoor behaviours that affect actual exposure). However, they note, MS prevalence is almost as strongly correlated with latitude per se, so the possibility that latitude and UVR are markers for some other geographically variant factor cannot be dismissed. Here, again, we glimpse the protean aetiological possibilities for MS. In the second paper, Axelson and colleagues from Sweden point out that the published literature (well, one paper) implicates geomagnetic latitude more strongly than geographic latitude.

That Swedish paper, based on a case-control analysis, reports a moderately strong association of MS with a selfreported history of exposure to ionising radiation (to be distinguished here from non-ionising UVR). Case-control studies using self-report exposure information are rather prone to bias because of differential recall or exposure by cases versus controls. These authors argue, however, that radiation exposure was not under-reported in their controls (although this would not preclude over-reporting in the cases). Confounding is the other major problem in case-control studies, and in this study there has been no serious attempt to control it. The strong, well-described association of MS with glandular fever, for example, might be associated with several of the sources of radiation examined. Interestingly, the idiom of this paper differs clearly from that of the companion paper, in that it views MS principally as the result of tissue damage caused by some exogenous exposure. This is a classic 'toxicological' model of MS. The authors, having observed an association with ionising radiation, postulate a central role for oxidative activity as the process that damages molecular myelin. Ionising radiation, as with certain biochemical exposures, increases the level of free radical activity and hence of oxidative damage to tissues. Hence they suggest ambient UVR level may act as a proxy for the level of ionising cosmic radiation (associated with geomagnetic polarity), which, too, may act via the induction of oxidative damage.

As we come to understand more about the ubiquity of viral infections and the complexity and developmental lability of the immune system, it becomes less likely that diseases such as MS will be explicable in these classic exposure-disease terms. Meanwhile, elucidating how viral infection, the immune response to it, and its modulation by other factors results in an autoimmune disease process presents other challenges to researchers.

For a start, we need to know if patterns of childhood and adolescent infection are correlated with - and therefore confound geographical analyses of - ambient levels of UVR. We need better understanding of whether and how UVR affects the human immune response: do immune indices vary by latitude, season, or indoor/outdoor occupation difference? It will be necessary to distinguish UVR from the visible light spectrum. As van der Mei and colleagues point out, there is some evidence that melatonin is immune-suppressive; melatonin levels vary in response to daylight levels.

Assuming that UVR does modulate immune activity, does it act in early life, influencing the initiating of the autoimmune process - or maybe even having a more general influence on the maturation of the young immune system? It is now clear, in mice, that UVR can influence Th $1 / 2$ balance. The plasticity of the immune system in the first year or two of life is also apparent in the manner in which the pattern of infections at this age determines subsequent atopy. Separating such an early-life effect from subsequent adult exposure may be difficult unless migrant studies are again used. A second possibility is that the UVR has an effect actually at the time when the triggering infection occurs. Should you sit in bed or on the beach with your glandular fever? Finally of course, UVR may modulate the intensity of immune and autoimmune activity in adulthood - in which case epidemiologists should seek evidence of a UVR effect on the rate of MS progression. (Indeed, among cases the age at death may vary as a function of adult UVR exposure.) As van der Mei and colleagues point out, experimental studies in mice show that UVR levels, vitamin D injections, light intensity and melatonin blocking variously affect all stages of experimental allergic encephalomyelitis, a wellrecognised analogue of MS in humans. The first step is undoubtedly to show that there is an association between ultraviolet exposure and MS at the individual level when confounding is adequately controlled. If so, we can then focus down on the precise mechanism and age of action.

A general model of autoimmune aetiology is emerging. Immune system susceptibility is determined both by a genetic component - frequently through HLA genes but also it appears now cytokine genes - and by an environmental component, largely determined by early-life exposures when the system is plastic, and including UVR exposure, infections and diet. This susceptible system is 
then primed, probably via the phenomenon of molecular mimicry, by an infection - in the case of MS by EpsteinBarr virus in adolescence and/or other viruses. Finally there is a proximal trigger - an event that again distorts normal immune response - perhaps a stressful life event or UVR again.

\section{References}

1 McMichael AJ, Hall AJ: Does immunosuppressive ultraviolet radiation explain the latitude gradient for multiple sclerosis? Epidemiology 1997;8:642-645.

2 Dumas M, Jauberteau-Marchan MO: The protective role of Langerhans' cells and sunlight in multiple sclerosis. Med Hypotheses 2000;55: 517-520.
This model is complex, but is highly testable at each of its steps and as a whole. A large-scale, multicentre casecontrol study of MS appears the logical next step.
3 Hernan MA, Zhang SM, Lipworth L, Olek MJ, Ascherio A: Multiple sclerosis and age at infection with common viruses. Epidemiology 2001;12:301-306.

4 Wandinger K, Jabs W, Siekhaus A, et al.: Association between clinical disease activity and Epstein-Barr virus reactivation in MS. Neurology 2000;55:178-184.
5 Ascherio A, Munch M: Epstein-Barr virus and multiple sclerosis. Epidemiology 2000;11:220224.

6 Fukazawa T, Yabe I, Kikuchi S, Sasaki H, Hamada T, Miyasaki K, Tashiro K: An association of vitamin $\mathrm{D}$ receptor gene polymorphism with multiple sclerosis in Japanese. J Neurol Sci 1999;166:47-52. 